\title{
Special Topic: Nanobiosensing
}

\author{
Huangxian $\mathrm{Ju}^{1}$
}

Published online: 14 July 2017

(C) The Nonferrous Metals Society of China and Springer Nature Singapore Pte Ltd. 2017

The 21 st century is witnessing rapid progress in life science and nanoscience. The unique properties of nanomaterials have made them effective platforms as electronic and optical signal transductions for the design of a new generation of biosensing devices. These properties and their synergic effect among catalytic activity, conductivity, and biocompatibility accelerate the signal transduction and amplification, which leads to lower detection limits and wider range of targets. These biosensing devices open up the novel concepts for the basic research and new tools for ultrasensitive in vitro or in vivo detection of biomolecules, cells, virus, and bacteria. The high sensitivity makes it possible to detect undetectable targets by the traditional methods, such as some disease markers, biological threat agents, and pathogens. Nanobiosensing, therefore, is increasingly important not only in scientific research, particularly analytical chemistry, but also in medicine, food processing industry, environment, security, and our daily lives, and has become one of the hottest topics in both life science and nanoscience, which expands the tentacles of bioanalysis and promotes the overall development of analytical science.

The development of nanobiosensing devices requires highly collaborative interactions between chemists, life scientists, and experts in nanotechnology as well as those in the fields of biomaterials, bioinformatics, and the physical sciences. The successful development and application of nanobiosensing technology are the results of the

Huangxian Ju

hxju@nju.edu.cn

1 State Key Laboratory of Analytical Chemistry for Life Science, Department of Chemistry, Nanjing University, Nanjing 210023, People's Republic of China fundamental understanding and study of confinement and orientation of biomacromolecules (e.g., proteins, peptides, and DNA) at micro- and nanointerfaces, the emerging of biorecognition strategies based on artificial recognition elements and synthetic materials, and the establishment of signal extraction technologies based on biological interactions. The bottleneck of this field should be the design of specific nanoprobes along with the signal switch and amplification strategies. The research topics in nanobiosensing involve fundamental works on highly sensitive and discriminative biosensing methods, including new approaches to integrated sensor systems, nanoprobe development, and actuators; novel, robust, easy to operate sensor systems with highly selective responses to multiple analytes under variable conditions, with increased sensitivity and significantly fewer false-positive and false-negative responses; innovative ideas in the development of novel biorecognition strategies; and development of multifunctional nanomaterials with predefined physical, chemical, or biological characteristics for biosensing applications.

Journal of Analysis and Testing is a new journal to provide an international academic platform for the publication of original research papers, rapid communications, and critical reviews in all aspects of fundamental and applied analytical chemistry. This special issue focusing on the topic "NanoBiosensing" includes but is not limited to biosensors, electrochemical biosensors, nanobiosensing, molecular recognition, imaging analysis, nanobiosensing devices, and nanoprobe. The first work, contributed by Professor Quan Cheng at the University of California, Riverside (USA), describes the tunable enhancement of electrospun nanofibers of graphene/polyaniline/poly (ethylene oxide) composite, which leads to sensitive response to methanol, ethanol, and 1-propanol vapors for their identification. The second paper from Professor Bin Su at Zhejiang University summarizes a 
newly emerging imaging technique based on electrogenerated chemiluminescence, which is being developed for detection of a wide range of analytes. Professor Serge Consier at Universite Joseph Fourier (France) then reports an original interfacial hybrid cross-linker molecule combined electrochemical and photochemical properties by substitution of two functional groups; Professor Chen-Zhong Li at Florida International University (USA) contributes a review to describe the detection of $\beta$-Amyloid biomarker for Alzheimer's disease; Professor Jun-Jie Zhu at Nanjing University describes the properties, synthesis, functionalization, and biosensing application of fluorescent gold nanoclusters; and Professor Yang Tian at East China Normal University reports an ultrasensitive and selective probe for ratiometric determination and removal of mercury ion. Finally, Professor Hui Chen at Fudan University studies the inclusion of naproxen in $\beta$-cyclodextrin and its release from the inclusion complex. This issue represents only a small sampling of the technical contributions from the nanobiosensing ocean. We hope that this special topic could provide valuable references to the related researchers.

We are grateful to all the authors who have shared our enthusiasm and vision by contributing high-quality manuscripts and keeping in tune with the theme of this issue. We also thank all referees for their careful reviewing of the submitted manuscripts. We deeply hope that analytical scientists will continue to contribute their excellent research studies for publication in Journal of Analysis and Testing.

Huangxian Ju

Professor in Chemistry

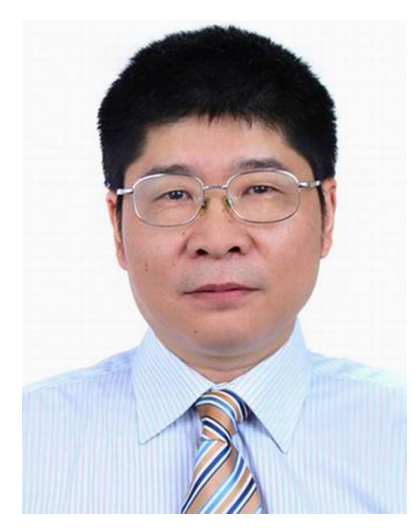

Huangxian Ju received his B.S., M.S., and Ph.D. degrees (1982-1992), and became an associate professor (1993) and professor (1999) in Nanjing University. He was a postdoctoral researcher in Montreal University (Canada) from 1996 to 1997. He won the National Funds for National Distinguished Young Scholars in 2003, and was selected as a Changjiang Professor in 2007, a "973" chief scientist in 2009, Fellows of the ISE and the Royal Society of Chemistry in 2015. He is the director of State Key Laboratory of Analytical Chemistry for Life Science. His research interest includes analytical biochemistry and nanobiosensing, focusing on signal amplification and life analytical chemistry. He has published 582 papers with an h-index of 80 (Google scholar h-index 88). 